\title{
Association between BRAF (V600E) mutation and clinicopathological features of papillary thyroid carcinoma: a Brazilian single-centre case series
}

Danielle Pessôa-Pereira', Mateus Fernandes da Silva Medeiros', Virna Mendonça Sampaio Lima', Joaquim Custódio da Silva Jr.' , Taíse Lima de Oliveira Cerqueira', Igor Campos da Silva², Luciano Espinheira Fonseca Jr. 2,3, Luiz José Lobão Sampaio4, Cláudio Rogério Alves de Lima ${ }^{5}$, Helton Estrela Ramos ${ }^{1}$

\begin{abstract}
Objectives: We aimed to investigate the prevalence of the BRAF (V600E) mutation in consecutive cases of papillary thyroid carcinoma (PTC) in patients diagnosed and treated at the Hospital Sao Rafael (Salvador, BA, Brazil) and evaluate its association with clinical and pathological characteristics of PTC. Subjects and methods: We retrospectively enrolled in the study a total of 43 consecutive PTC patients who underwent total thyroidectomy. We performed DNA extraction from formalinfixed paraffin-embedded (FFPE) tumour tissue samples. Polymerase chain reaction (PCR) and direct sequencing were used to determine BRAF (V600E) mutation status. Univariate and multivariate logistic regression analyses were employed to identify independent associations. Results: The prevalence of BRAF (V600E) mutation was $65.1 \%$ (28/43). A high frequency of older patients ( $p$ value: 0.004 ) was observed among the BRAF-mutated PTC group and, in contrast, a low frequency of concurrent Hashimoto's thyroiditis (HT) ( $p$ value: 0.011 ) was noted. Multivariate analysis confirmed that older age (OR: 1.15; 95\% Cl: 1.00 - 1.33; p value: 0.047 ) and HT (OR: 0.05; 95\% Cl: 0.006-0.40; $p$ value: 0.005$)$ were independent factors associated with $B R A F(\mathrm{~V} 600 \mathrm{E})$ mutation. Conclusion: We found a high prevalence of BRAF (V600E) mutation in PTC cases. Older age and no concurrent HT were independently associated with BRAF (V600E) mutation. Arch Endocrinol Metab. 2019;63(2):97-106
\end{abstract}

Keywords

Papillary thyroid cancer; BRAF mutation; Hashimoto's thyroiditis
${ }^{1}$ Departamento de Biorregulação, Laboratório de Estudo da Tireoide, Instituto de Saúde e Ciência, Universidade Federal da Bahia (UFBA), Salvador, BA, Brasil ${ }^{2}$ Departamento de Anatomia Patológica e Citopatologia, Hospital São Rafael, Salvador, BA, Brasi ${ }^{3}$ Departamento de Anatomia Patológica e Medicina Legal, Faculdade de Medicina da Bahia Universidade Federal da Bahia (UFBA), Salvador, BA, Brasil

${ }^{4}$ Departamento de Medicina Nuclear, Hospital São Rafael, Salvador, BA, Brasil

${ }^{5}$ Departamento de Cirurgia de Cabeça e Pescoço, Hospital São Rafael, Salvador, BA, Brasil

Correspondence to: Helton Estrela Ramos Departamento de Biorregulação, Universidade Federal da Bahia Av. Reitor Miguel Calmon, $s / n$, Vale da Canela, sala 300 40110-100 - Salvador, BA, Brasil ramoshelton@gmail.com

Received on Nov/20/2018 Accepted on Jan/14/2019

DOI: 10.20945/2359-3997000000120

\section{INTRODUCTION}

$\mathrm{T}$ hyroid cancer is the most frequently diagnosed endocrine malignancy worldwide, currently ranking in ninth place for global incidence (1). Papillary thyroid carcinoma (PTC), which accounts for up to $85 \%$ of all thyroid cancer cases (2), has a relatively indolent behaviour and shows better prognosis than other malignant thyroid tumours, such as medullary and anaplastic thyroid carcinoma (3). However, some patients may experience extrathyroidal extension (ETE), local and/or distant metastases, as well as present recurrent disease after surgery and radioactive iodine therapy, all of which significantly contribute to a poorer prognosis (4). Recurrence and mortality risk stratifications, which are mainly based on clinical and pathological criteria, are currently the main tool for determining suitable clinical management of PTC $(5,6)$. Nevertheless, those clinicopathological factors, such as older age, histological subtypes, tumour size and distant metastasis, have not been able to provide complete accuracy in terms of predicting a poor prognosis (7). Therefore, many studies have focused on identifying additional parameters, such as molecular markers, in order to provide an accurate risk assessment.

Significant advances in understanding oncogenic events involved in the onset and progression of PTC have arisen over recent decades. Thyroid oncogenesis 
often involves constitutive activation of the mitogenactivated protein kinase (MAPK) kinase (MEKK)/ extracellular signal-regulated kinase (ERK) pathway, usually driven by the T1799A somatic mutation in the v-raf murine sarcoma viral oncogene homolog $\mathrm{Bl}$ $(B R A F)$ exon 15 , a process which results in a $\mathrm{V} 600 \mathrm{E}$ amino acid replacement (8). Several studies have shown a positive association between $B R A F$ (V600E) and PTC aggressive phenotype, including ETE, distant metastasis, and silencing of thyroid-specific iodinemetabolizing genes (9). On the other hand, these findings have not been found in some other studies (10), raising doubts about whether BRAF (V600E) could be used as an appropriate prognostic factor for PTC.

Considering the lack of consistent evidence in the literature and that there are no published reports on the prevalence of $B R A F(\mathrm{~V} 600 \mathrm{E})$ mutation in PTC patients in North-eastern Brazil, we conducted a crosssectional study of consecutive case series of PTC from a reference hospital in Salvador, BA, Brazil, in order to evaluate the association between $B R A F(\mathrm{~V} 600 \mathrm{E})$ mutation and clinicopathological features of PTC.

\section{SUBJECTS AND METHODS}

\section{Ethics statement}

This study was formally approved by the institutional Research Ethics Committee of the Federal University of Bahia (n. 102.290), along with the medical board of Sao Rafael Hospital (Salvador, Bahia, Brazil), and was carried out in accordance with the Declaration of Helsinki of the World Medical Association.

\section{Patient selection}

We retrospectively enrolled 43 consecutive PTC patients who had undergone total thyroidectomy as initial treatment for PTC in the Department of Head and Neck Surgery at Sao Rafael Hospital between 2006 and 2012. All clinical and pathological data, including age, sex, tumour size, histological subtype, multifocality, concurrent Hashimoto's thyroiditis (HT), ETE, vascular invasion, lymph node and distant metastasis status, were collected from medical records (Table 1). Patients were classified as low, intermediate and high risk according to the 2015 American Thyroid Association (ATA) risk stratification for well-differentiated thyroid cancer $(6,11)$, as well as according to the Brazilian consensus (5). We excluded from the study patients whose cancer-related medical records and/or formalinfixed paraffin-embedded (FFPE) tumour specimens derived from their surgical resections were unavailable.

\section{Tumor samples}

FFPE blocks were obtained from the archives of the Department of Anatomic Pathology and Cytopathology at Sao Rafael Hospital in order to acquire thyroid tumour samples for BRAF (V600E) mutation analysis. All samples were histologically reviewed on haematoxylin and eosin (HE)-stained slides by two pathologists. Tumours were staged according to the $8^{\text {th }}$ edition of the TNM-based staging system proposed by the American Joint Association on Cancer (AJCC). Representative tumour areas containing at least $60 \%$ of cancer cells were marked on the HE-stained sections. Subsequently, five sequential sections of $10-\mu \mathrm{m}$ thickness were obtained from each chosen FFPE tissue block. Marked tumour areas were manually scraped off from the unstained sections by sterile needles using their respective HE-stained slides as a guide. In cases of multifocal disease, we only collected the largest tumour focus for analysis.

\section{DNA isolation}

Genomic DNA isolation from the FFPE samples was performed using Gentra Puregene Core Kit B (Qiagen, Valencia, CA, USA) at the Thyroid Study Laboratory (Salvador, BA, Brasil), preceded by a total removal of the paraffin from the samples with xylene and washing steps with ethanol, according to the manufacturer's protocol. Concentration levels and quality were assessed using a Nano Spectrophotometer Kasvi K230002 (Kasvi, Sao Jose dos Pinhais, PR, Brazil).

\section{Polymerase chain reaction}

Polymerase chain reaction (PCR) was performed to amplify the exon 15 of $B R A F$ from the isolated DNA, as previously described (8). Briefly, BRAF exon 15 was amplified in a $20-\mu \mathrm{l}$ reaction volume containing $100 \mathrm{ng}$ of genomic DNA, 7.5 pmol of each primer (Forward: 5' - AAACTCTTCATAATGCTTGCTCTG-3'; Reverse: 5'-GGCCAAAAATTAATCAGTGGA-3'), $100 \mu \mathrm{m}$ deoxynucleoside triphosphates (dNTPs), 5 $\mu \mathrm{Ci}\left[\alpha^{32} \mathrm{P}\right] \mathrm{dCTP}, 1.5 \mathrm{~mm} \mathrm{MgCl}_{2}$, Platinum TaqDNA polymerase high fidelity and buffer (Thermo Fisher Scientific, Waltham, MA, USA). The PCR reaction was 
Table 1. Clinical and pathological features of the PTC patients

\begin{tabular}{|c|c|c|c|c|c|c|c|c|c|c|c|c|c|c|c|c|}
\hline \multirow{2}{*}{ Patient } & \multirow{2}{*}{$\begin{array}{c}\text { Age } \\
(y)\end{array}$} & \multirow{2}{*}{ Sex } & \multirow{2}{*}{$\begin{array}{l}\text { Tumour } \\
\text { size (mm) }\end{array}$} & \multirow{2}{*}{ PTC variant } & \multirow{2}{*}{ HT } & \multirow{2}{*}{$\begin{array}{l}\text { Multifocal } \\
\text { disease }\end{array}$} & \multirow{2}{*}{ ETE } & \multirow{2}{*}{$\begin{array}{l}\text { Vascular } \\
\text { invasion }\end{array}$} & \multirow{2}{*}{ LM } & \multirow{2}{*}{$\begin{array}{c}\text { Distant } \\
\text { metastasis }\end{array}$} & \multicolumn{4}{|c|}{ 8th AJCC TNM } & \multirow{2}{*}{$\begin{array}{c}2015 \text { ATA } \\
\text { Recurrence Risk }\end{array}$} & \multirow{2}{*}{$\begin{array}{c}\text { BRAF (V600E) } \\
\text { mutation }\end{array}$} \\
\hline & & & & & & & & & & & $T$ & $\mathbf{N}$ & & $\overline{C S}$ & & \\
\hline 1 & 20 & $\mathrm{~F}$ & 25 & Classical & Yes & Yes & No & Yes & No & No & 2 & 0 & 0 & I & Intermediate & Negative \\
\hline 2 & 12 & $\mathrm{~F}$ & 40 & Classical & Yes & Yes & No & No & Yes & No & 2 & $1 b$ & 0 & I & High & Negative \\
\hline 3 & 17 & $\mathrm{~F}$ & 6 & Classical & No & No & No & No & Yes & No & $1 a$ & $1 \mathrm{~b}$ & 0 & । & Intermediate & Negative \\
\hline 4 & 17 & $\mathrm{~F}$ & 10 & Classical & No & No & No & No & No & No & $1 \mathrm{a}$ & 0 & 0 & I & Low & Positive \\
\hline 5 & 13 & $\mathrm{~F}$ & 22 & Classical & Yes & Yes & No & No & No & No & 2 & 0 & 0 & I & Low & Negative \\
\hline 6 & 36 & $\mathrm{~F}$ & 25 & Follicular & Yes & Yes & No & No & No & No & 2 & 0 & 0 & I & Low & Negative \\
\hline 7 & 40 & $\mathrm{~F}$ & 20 & Follicular & No & Yes & No & No & No & Yes & $1 b$ & 0 & 1 & $\|$ & High & Positive \\
\hline 8 & 26 & $\mathrm{~F}$ & 15 & Classical & Yes & Yes & Yes & No & Yes & No & $1 \mathrm{a}$ & $1 b$ & 0 & I & Intermediate & Positive \\
\hline 9 & 44 & $F$ & 10 & Classical & Yes & No & No & No & Yes & No & $1 a$ & 1a & 0 & 1 & Low & Negative \\
\hline 10 & 38 & $\mathrm{~F}$ & 12 & Classical & Yes & Yes & No & No & No & No & $1 b$ & 0 & 0 & 1 & Low & Positive \\
\hline 11 & 39 & F & 12 & Classical & Yes & Yes & No & No & No & No & $1 b$ & 0 & 0 & 1 & Low & Negative \\
\hline 12 & 26 & $\mathrm{~F}$ & 10 & Classical & No & No & No & No & Yes & No & $1 a$ & $1 \mathrm{a}$ & 0 & I & Low & Positive \\
\hline 13 & 40 & M & 13 & Classical & No & Yes & No & No & No & No & $1 b$ & 0 & 0 & I & Low & Positive \\
\hline 14 & 31 & $\mathrm{~F}$ & 30 & Follicular & No & No & No & No & No & No & 2 & 0 & 0 & I & Low & Negative \\
\hline 15 & 45 & $\mathrm{~F}$ & 15 & Classical & No & No & Yes & No & Yes & No & $1 b$ & $1 \mathrm{a}$ & 0 & 1 & Intermediate & Positive \\
\hline 16 & 36 & $F$ & 10 & Follicular & No & No & No & No & Yes & No & $1 a$ & $1 \mathrm{a}$ & 0 & 1 & Low & Negative \\
\hline 17 & 41 & $\mathrm{~F}$ & 5 & Classical & Yes & Yes & No & No & Yes & No & $1 \mathrm{a}$ & 1a & 1 & 1 & Low & Negative \\
\hline 18 & 45 & $\mathrm{~F}$ & 20 & Classical & No & Yes & No & No & No & No & $1 b$ & 0 & 0 & I & Low & Positive \\
\hline 19 & 32 & $\mathrm{~F}$ & 16 & Classical & Yes & No & No & No & No & No & $1 b$ & 0 & 0 & I & Low & Negative \\
\hline 20 & 36 & $\mathrm{~F}$ & 13 & Follicular & No & No & No & No & No & Yes & $1 b$ & 0 & 1 & $\|$ & High & Negative \\
\hline 21 & 35 & $\mathrm{~F}$ & 15 & Tall cell & Yes & No & No & No & Yes & No & $1 b$ & $1 \mathrm{a}$ & 0 & I & High & Negative \\
\hline 22 & 30 & $\mathrm{~F}$ & 15 & Follicular & No & Yes & No & No & Yes & No & $1 b$ & $1 \mathrm{a}$ & 0 & I & Intermediate & Negative \\
\hline 23 & 24 & $\mathrm{~F}$ & 10 & Classical & No & No & No & No & No & No & $1 a$ & 0 & 0 & 1 & High & Positive \\
\hline 24 & 37 & $\mathrm{~F}$ & 20 & Classical & No & Yes & No & No & No & No & $1 b$ & 0 & 0 & 1 & High & Positive \\
\hline 25 & 42 & M & 15 & Classical & No & No & No & No & No & No & $1 b$ & 0 & 0 & 1 & Low & Positive \\
\hline 26 & 33 & $\mathrm{~F}$ & 20 & Classical & Yes & No & Yes & No & Yes & No & $3 b$ & $1 \mathrm{a}$ & 0 & I & High & Positive \\
\hline 27 & 29 & $F$ & 10 & Classical & No & No & No & No & No & No & $1 a$ & 0 & 0 & 1 & Low & Positive \\
\hline 28 & 27 & $\mathrm{~F}$ & 20 & Classical & No & Yes & No & No & No & No & $1 b$ & 0 & 0 & I & Low & Positive \\
\hline 29 & 24 & $\mathrm{~F}$ & 20 & Classical & Yes & No & No & No & No & No & $1 b$ & 0 & 0 & 1 & Low & Negative \\
\hline 30 & 41 & M & 35 & Classical & No & No & No & No & No & No & 2 & 0 & 0 & 1 & Low & Positive \\
\hline 31 & 46 & $\mathrm{~F}$ & 10 & Follicular & No & Yes & No & No & Yes & No & $1 \mathrm{a}$ & 1a & 0 & 1 & Low & Positive \\
\hline 32 & 46 & $\mathrm{~F}$ & 37 & Classical & No & Yes & No & Yes & Yes & No & 2 & $1 \mathrm{a}$ & 0 & 1 & Intermediate & Positive \\
\hline 33 & 46 & $\mathrm{~F}$ & 9 & Classical & No & Yes & No & No & No & No & $1 a$ & 0 & 0 & I & Low & Positive \\
\hline 34 & 61 & $F$ & 50 & Trabecular & No & Yes & No & No & No & No & $3 a$ & 0 & 0 & $\|$ & Low & Positive \\
\hline 35 & 42 & F & 30 & Classical & Yes & No & No & No & Yes & No & 2 & $1 b$ & 0 & I & Low & Positive \\
\hline 36 & 62 & $\mathrm{~F}$ & 50 & Classical & Yes & Yes & No & Yes & No & No & $3 a$ & 0 & 0 & $\|$ & Intermediate & Positive \\
\hline 37 & 44 & F & 15 & Oncocytic & No & Yes & No & No & No & No & $1 b$ & 0 & 0 & I & Low & Positive \\
\hline 38 & 28 & $\mathrm{~F}$ & 15 & Classical & No & No & No & No & No & No & $1 b$ & 0 & 0 & 1 & Low & Positive \\
\hline 39 & 41 & $\mathrm{~F}$ & 13 & Classical & No & Yes & No & No & Yes & No & $1 b$ & $1 \mathrm{a}$ & 0 & 1 & Low & Positive \\
\hline 40 & 36 & $\mathrm{~F}$ & 13 & Classical & No & No & No & No & No & No & $1 b$ & 0 & 0 & I & Low & Positive \\
\hline 41 & 78 & $\mathrm{~F}$ & 15 & Follicular & No & Yes & No & No & No & No & $1 b$ & 0 & 0 & 1 & Low & Positive \\
\hline 42 & 69 & $\mathrm{~F}$ & 18 & Classical & Yes & Yes & Yes & No & No & No & $3 b$ & 0 & 0 & $\|$ & High & Positive \\
\hline 43 & 38 & $\mathrm{~F}$ & 18 & Classical & Yes & No & No & No & Yes & No & $1 b$ & 1a & 0 & 1 & High & Positive \\
\hline
\end{tabular}

Y: years-old; F: female; M: male; mm: millimetres; PTC: papillary thyroid carcinoma; HT: Hashimoto's thyroiditis; ETE: extrathyroidal extension; AJCC: American Joint Committee on Cancer; CS: clinical stage; ATA: American Thyroid Association. 
heated to $95{ }^{\circ} \mathrm{C}$ for 5 minutes for initial denaturation followed by 35 cycles of $95{ }^{\circ} \mathrm{C}$ for 30 seconds, $58{ }^{\circ} \mathrm{C}$ for 30 seconds and $72{ }^{\circ} \mathrm{C}$ for 45 seconds, with a final extension at $72^{\circ} \mathrm{C}$ for 5 minutes. All PCR amplifications were performed using the Veriti 96-Well Thermal Cycler (Thermo Fisher Scientific, Waltham, MA, USA).

\section{Agarose gel eletrophoresis}

PCR products were electrophoresed in 1,5\% UltraPure ${ }^{\mathrm{TM}}$ Agarose diluted in UltraPure ${ }^{\mathrm{TM}}$ TBE Buffer IX (Thermo Fisher Scientific, Waltham, MA, USA) containing 0,1$\mu \mathrm{l} / \mathrm{mL}$ SYBR Safe DNA Gel Stain (Thermo Fisher Scientific, Waltham, MA, USA) at 90v for 45 minutes using the Loccus horizontal gel electrophoresis system, model LCH 13x15 (Loccus Biotechnology, Cotia, SP, Brazil). BRAF exon 15 amplification was confirmed by the presence of single bands containing 231 base pairs visualized using a Safe Imager 2.0 (Thermo Fisher Scientific, Waltham, MA, USA).

\section{DNA sequecing}

After confirmation of BRAFexon 15 amplification, PCR products were purified using the PureLink Quick PCR Purification Kit (Thermo Fisher Scientific, Waltham, MA, USA) and precipitated using 70\% isopropyl alcohol and Hi-DITM Formamide (Thermo Fisher Scientific, Waltham, MA, USA). Subsequently, DNA sequencing reaction were performed using a BigDye ${ }^{\circledR}$ Terminator v3.1 Cycle Sequencing Kit (Thermo Fisher Scientific, Waltham, MA, USA) in the capillary automatic sequencer ABI 3130XL PRISM Genetic Analyzer (Thermo Fisher Scientific, Waltham, MA, USA) at the Fundação Oswaldo Cruz - Instituto Gonçalo Moniz (Salvador, Bahia, Brazil). The BRAF (V600E) mutation was confirmed by comparing sequences using the Basic Local Alignment Search Tool (BLAST) program, available at the NCBI website (http://www. ncbi.nlm.nih.gov/BLAST/). We considered tumours as being $B R A F(\mathrm{~V} 600 \mathrm{E})$ positive those which were harbouring a homozygous or heterozygous $B R A F$ (V600E) mutation (T1799A) (Figure 1).

\section{Statistical analysis}

Qualitative variables were presented using frequencies and percentages, whereas continuous variables were presented using mean values and standard deviation. An independent $t$-test was used to compare the mean values of continuous variables and a chi-square test or Fisher's exact test to compare frequencies of qualitative variables. Univariate and multivariate logistic regression

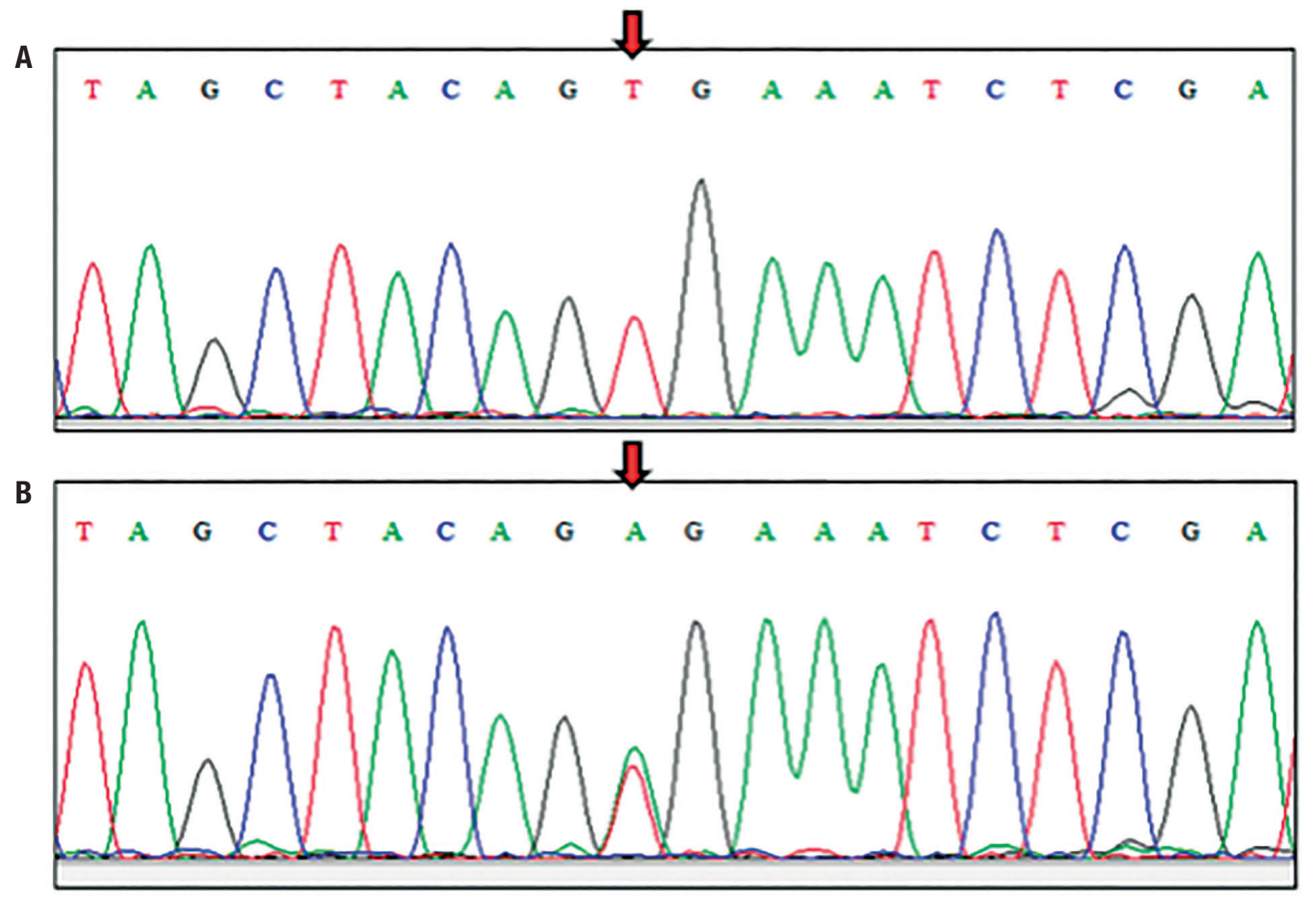

Figure 1. Representative DNA sequencing of BRAF exon 15. A) Absence of thymine to adenine replacement (T1799A), indicating a wild-type BRAF. B) Heterozygous BRAF (V600E) mutation. 
analyses were performed to evaluate the associations between clinical and pathological parameters of PTC and the BRAF (V600E) mutation. Values of odds ratio $(\mathrm{OR})$ were determined to measure the strength of the associations. The statistical significance of these associations was based on $\mathrm{p}$ value $<0.05$ and $95 \%$ confidence intervals (CI). All statistical data analyses were performed using the statistical software program SPSS for Windows, v. 20.0 (IBM, Armonk, NY, USA).

\section{RESULTS}

\section{Clinical and pathological characteristics of PTC patients}

All clinical and pathological data of the 43 selected PTC patients are summarized in Table 2 . The mean age at diagnosis was 37 years, ranging from 12 to 78 years. Even though only four patients $(3.6 \%)$ were considered as paediatric cases $(\leq 18$ years old), most patients were $>55$ years old $(90.7 \%)$. Therefore, our case series were mainly comprised by early-stage PTC patients $(88.4 \%$ and $11.6 \%$ for clinical stage I and II, respectively), according to current pathological TNM system. Most patients were female $(40 / 43 ; 93 \%)$ and presented multifocal disease $(23 / 43 ; 53.5 \%)$. Seventeen patients (39.5\%) had concurrent Hashimoto's thyroiditis (HT). As expected, there were more conventional PTC cases $(32 / 43 ; 8 / 43 ; 74.4 \%)$, followed by the follicular variant $(8 / 43 ; 18.6 \%)$. Three cases of rare PTC histological subtypes were observed, which included an oncocytic (oxyphilic), variant, a trabecular variant and a tall cell variant. Tumour sizes ranged from 5 to $50 \mathrm{~mm}$ (mean, $19.23 \mathrm{~mm}$ ), most of them having more than $10 \mathrm{~mm}(32 / 43 ; 74.4 \%)$. Furthermore, 16 patients $(37.2 \%)$ had lymph node metastasis at diagnosis, but only few cases presented vascular invasion $(3 / 43 ; 7 \%)$, ETE $(4 / 43 ; 9.3 \%)$ and distant metastases $(2 / 43 ; 2 \%)$. As stated by 2015 ATA risk stratification, 27 (62.8\%) and 16 patients had low and intermediate/high risk of recurrence, respectively. Moreover, 24 (55.8\%) and 19 (44.2\%) patients had very low/low and intermediate/ high risk of recurrence, respectively, according to the current Brazilian consensus.

\section{Association of BRAF (V600E) mutation and clinicopathological characteristics of PTC}

$B R A F$ (V600E) mutation was detected in 28 PTC tumour samples $(65.1 \%)$. No homozygous mutant or other genetic alterations were found in $B R A F$ exon 15 . Patients were categorized according to $B R A F$ (V600E) mutation status in order to evaluate possible associations between clinical and pathological features of PTC and presence of $B R A F(\mathrm{~V} 600 \mathrm{E})$ (Table 2). All $\geq 55$-yearold $(4 / 43)$ and male $(3 / 43)$ patients presented PTC harbouring a BRAF (V600E) mutation. Only one paediatric patient $(1 / 3)$ had a $B R A F$-mutant PTC: a 17-year-old young woman who presented PTC with no aggressive behaviour. Furthermore, follicular variant was predominantly observed in the $B R A F$-wild type PTC group $(5 / 8 ; 62.5 \%)$. BRAF (V600E) was detected in the isolated rare cases of trabecular variant and oncocytic variant, but not in the tall cell PTC variant. All patients who presented PTC with ETE (4/43) were tested as $B R A F$ (V600E) positives.

After comparing the groups, we found that the frequency of BRAF (V600E) mutation was significantly higher in older patients $(40.96 \pm 13.69$ vs $29.73 \pm 10.18$; p value: 0.004 ) and lower in PTC with concurrent HT ( 25 vs $66.7 \%$; p value: 0.011 ). However, no significant differences were found for other clinical and pathological parameters, including intermediate to high risk of recurrence.

\section{Univariate and multivariate logistic regression analysis}

To further investigate the effect of BRAF (V600E) on age and HT in PTC patients, we performed a univariate analysis to measure the association between these parameters and the presence of a $B R A F(\mathrm{~V} 600 \mathrm{E})$ mutation (Table 3 ). Once again, we found that older age at diagnosis (OR: 1.09; 95\% CI: 1.02-1.17; p value: 0.016$)$ and negative $\mathrm{HT}$ (OR: $0.17 ; 95 \% \mathrm{CI}$ : $0,04-0,66 ; p$ value: 0.011$)$ were significantly associated with $B R A F$-mutated PTC. Finally, we performed a multivariate regression logistic analysis in order to investigate whether these features could be considered as independent predictors of $B R A F(\mathrm{~V} 600 \mathrm{E})$ mutation. Crude $\mathrm{OR}$ values confirmed the significant associations revealed in the univariate analysis. After adjusting for tumour size, multifocality, vascular invasion, extrathyroidal extension, lymph node and distant metastasis, we found that older age (OR: 1.15; 95\% CI: $1.00-1.33$; p value: 0.047 ) and negative HT (OR: 0.05; 95\% CI: $0.006-0.40$; p value: 0.005$)$ were still significantly associated with the BRAF (V600E) mutation. 
Table 2. Associations between BRAF (V600E) mutation and clinicopathological features of PTC

\begin{tabular}{|c|c|c|c|c|}
\hline Characteristics & All patients & BRAF mutation (-) & BRAF mutation (+) & $\mathrm{p}$ value \\
\hline Total number of patients (\%) & 43 & 15 & 28 & - \\
\hline Paediatric patients ( $\leq 18 \mathrm{y}$ ) & $4(9.3)$ & 3 & $1(3.6)$ & \\
\hline \multicolumn{5}{|l|}{ Age at diagnosis, y } \\
\hline Mean & $37.04 \pm 13.58$ & $29.73 \pm 10.18$ & $40.96 \pm 13.69$ & 0.004 \\
\hline Median & 37 & & & \\
\hline Range & $12-78$ & & & \\
\hline$<55$ & $39(90.7)$ & $15(100)$ & $24(85.7)$ & $0.280^{\mathrm{a}}$ \\
\hline$\geq 55$ & $4(9.3)$ & 0 & $4(14.3)$ & \\
\hline Sex & & & & $0.540^{\mathrm{a}}$ \\
\hline Male & $3(7)$ & 0 & $3(10.7)$ & \\
\hline Female & $40(93)$ & $15(100)$ & 25 (89.3) & \\
\hline \multicolumn{5}{|l|}{ Tumour size, mm } \\
\hline Mean & $19.23 \pm 11.36$ & $17.60 \pm 9.51$ & $19.21 \pm 11.20$ & 0.622 \\
\hline Median & 15 & & & \\
\hline Range & $5-50$ & & & \\
\hline$<10$ & $11(25.6)$ & $2(13.3)$ & $1(3.6)$ & $0.275^{\mathrm{a}}$ \\
\hline$\geq 10$ & $32(74.4)$ & $13(86.7)$ & 27 (96.4) & \\
\hline Histological subtype & & & & 0.150 \\
\hline Classical PTC & $32(74.4)$ & $9(60)$ & $23(82.1)$ & \\
\hline Non-classical PTC & $11(25.6)$ & $6(40)$ & $5(17.9)$ & \\
\hline Follicular variant & $8(18.6)$ & $5(33.3)$ & $3(10.7)$ & \\
\hline Tall cell variant & $1(3.6)$ & $1(6.7)$ & 0 & \\
\hline Trabecular variant & $1(3.6)$ & 0 & $1(3.6)$ & \\
\hline Oncocytic variant & $1(3.6)$ & 0 & $1(3.6)$ & \\
\hline Hashimoto's thyroiditis & & & & 0.011 \\
\hline No & $26(60.5)$ & $5(33.3)$ & $21(75)$ & \\
\hline Yes & $17(39.5)$ & $10(66.7)$ & $7(25)$ & \\
\hline Multifocal disease & & & & 0.54 \\
\hline No & $20(46.5)$ & $8(53.3)$ & $12(42.9)$ & \\
\hline Yes & $23(53.5)$ & $7(46.7)$ & $16(57.1)$ & \\
\hline ETE & & & & $0.280^{\mathrm{a}}$ \\
\hline No & $39(90.7)$ & $15(100)$ & $24(85.7)$ & \\
\hline Yes & $4(9.3)$ & 0 & $4(14.3)$ & \\
\hline Vascular invasion & & & & $1^{\mathrm{a}}$ \\
\hline No & $40(93)$ & $14(93.3)$ & 26 (92.9) & \\
\hline Yes & $3(7)$ & $1(6.7)$ & $2(7.1)$ & \\
\hline Lymph node metastasis & & & & 0.348 \\
\hline No & $27(62.8)$ & $8(53.3)$ & $19(67.9)$ & \\
\hline Yes & $16(37.2)$ & $7(46.7)$ & $9(32.1)$ & \\
\hline Distant metastasis & & & & $1^{\mathrm{a}}$ \\
\hline No & $41(95.3)$ & $14(93.3)$ & $27(96.4)$ & \\
\hline Yes & $2(4.7)$ & $1(6.7)$ & $1(3.6)$ & \\
\hline 8th AJCC/TNM stage system & & & & $0.643^{\mathrm{a}}$ \\
\hline I & $38(88.4)$ & $14(93.3)$ & $24(85.7)$ & \\
\hline$\|$ & $5(11.6)$ & $1(6.7)$ & $4(14.3)$ & \\
\hline 2015 ATA recurrence risk & & & & 0.782 \\
\hline Low & $27(62.8)$ & $9(60)$ & $18(64.3)$ & \\
\hline Intermediate/High & $16(37.2)$ & $6(40)$ & $10(35.7)$ & \\
\hline SBEM recurrence risk & & & & 1 \\
\hline Very low/Low & $24(55.8)$ & $9(60)$ & $16(57.1)$ & \\
\hline Intermediate/High & $19(44.2)$ & $6(40)$ & $12(42.9)$ & \\
\hline
\end{tabular}


Table 3. Univariate and multivariate regression logistic analysis of BRAF (V600E) mutation and clinicopathological features of PTC

\begin{tabular}{|c|c|c|c|c|c|c|}
\hline \multirow{2}{*}{ Characteristics } & \multicolumn{3}{|c|}{ Univariate } & \multicolumn{3}{|c|}{ Multivariate } \\
\hline & OR (95\% Cl) & $p$ value & OR (95\% Cl) & $p$ value & Adjusted OR ${ }^{\mathrm{a}}(95 \% \mathrm{Cl})$ & p value \\
\hline Age at diagnosis, y & 1 & & 1 & & 1 & \\
\hline Mean & $1.09(1.02-1.17)$ & 0.016 & $1.09(1.01-1.18)$ & 0.021 & $1.15(1.00-1.33)$ & 0.047 \\
\hline \multicolumn{7}{|c|}{ Hashimoto's thyroiditis } \\
\hline No & 1 & & 1 & & 1 & \\
\hline Yes & $0.17(0.04-0.66)$ & 0.011 & $0.14(0.03-0.68)$ & 0.014 & $0.05(0.006-0.40)$ & 0.005 \\
\hline
\end{tabular}

Y: years; OR: odds ratio.

a: Adjusted for tumour size, multifocality, vascular invasion, extrathyroidal extension, lymph node and distant metastasis.

\section{DISCUSSION}

Although PTC usually displays excellent behaviour at clinical presentation (3), some cases may present a worse prognosis, with ETE, lymph node and distant metastases, as well as acquiring treatment resistance (4). In an effort to find potential prognostic biomarkers in PTC that could identify those patients, several studies have investigated the role of the BRAF (V600E) mutation, a common genetic alteration in PTC. However, it remains unclear whether $B R A F$ (V600E) is closely associated with aggressive behaviour of PTC. Because few studies have been performed by using Brazilian cohorts, this research was designed to verify the frequency of $B R A F(\mathrm{~V} 600 \mathrm{E})$ mutation in PTC patients in a reference hospital in Salvador, BA, Brazil, and investigate its association with their clinical and pathological characteristics.

The prevalence rates of the $B R A F(\mathrm{~V} 600 \mathrm{E})$ mutation in PTC range between 27.3 to $90.2 \%$ worldwide $(12,13)$. Most Brazilian studies that investigated the presence of $B R A F$ mutation in FFPE or fresh frozen PTC samples were conducted in the South-eastern region, mainly in the State of Sao Paulo (14-18). While these studies reported $B R A F(\mathrm{~V} 600 \mathrm{E})$ frequencies between 28.1 to $48.3 \%$, we noted a higher prevalence rate $(65.1 \%)$ in PTC patients in the present study. Our finding is similar to those reported in PTC patients diagnosed in cities from other Brazilian regions, such as Goiânia (74/116; 63,8\%) and Porto Alegre (19/32; $59,4 \%)(19,20)$. Some studies have implied that the increased occurrence of $B R A F$ (V600E) mutation in PTC may be associated with high dietary iodine intake (21). We found in a previous study from our laboratory a high risk of excessive nutritional iodine intake among schoolchildren in Salvador (22), a coastal Brazilian city. If extrapolated to the adult population, this finding suggested that most residents in Salvador may also have a relatively high iodine intake. However, considering the low frequency reported by the studies conducted in Sao Paulo, where thyroid cancer incidence and iodine intake levels are considered higher than other Brazilian regions (23), we assume there are other features that could have influenced the high prevalence rate of $B R A F(\mathrm{~V} 600 \mathrm{E})$ mutation found in the present study, such as environmental factors and genetic background of the PTC patients.

In the past few decades, numerous meta-analyses have reported BRAF (V600E) as being strictly associated with aggressive clinicopathological features and poorer clinical outcomes PTC $(12,13)$, but this association remains controversial. We found that $B R A F$ (V600E) mutation was significantly linked with older age, with such patients being more likely to have a $B R A F$-mutated PTC according to univariate and multivariate analyses. Advanced age has been considered an important prognostic factor for PTC patients and is significantly associated with recurrence and survival (24). Interestingly, Shen and cols. have recently demonstrated in a large cohort that age is an independent risk factor for mortality in PTC patients with $B R A F$ (V600E) mutation, but not in wild type $B R A F$ (25). In contrast to the adult population, differentiated thyroid cancer is an uncommon event in childhood and adolescence (26). From our case series, only four PTC patients were classified as paediatric cases. As opposed to adult PTC, paediatric PTC usually displays aggressive behaviour at clinical presentation, manifested by high incidence of multifocal disease, lymph node and distant metastasis (26). In spite of that, paediatric PTC often has a better prognosis compared to adult PTC (26). Among all paediatric PTC patients enrolled in the present study, only one presented a BRAF-mutant PTC. Consistently, the literature has reported low frequency of $B R A F$ mutations in paediatric PTC (27). Conversely, rearranged during 
transfection (RET)/PTC translocations seems to be the most frequent genetic change in those cases, which are most often associated with radiation exposure (27). Remarkably, BRAF (V600E) mutation, but not RET/ PTC rearrangements, has been associated with genomic instability and consequently with decreased expression of the sodium-iodine symporter (NIS) (14,28), somewhat corroborating the better recurrence-free survival observed in paediatric PTC patients when compared to adults.

HT is an autoimmune disease that promotes inflammation and subsequently a progressive depletion of thyroid cells, which are gradually replaced by fibrosis and mononuclear infiltrate (29). Although the association between inflammation and cancer has been well established in distinct human cancers (30), the pathological and molecular connections between HT and PTC are still discussed. It has been postulated that genetic alterations promoting carcinogenesis can activate proinflammatory programs, which result in the formation of an inflammatory tumour microenvironment (31). In turn, the inflammatory cells contribute to tumour progression by inducing the expression of growth factors, proangiogenic factors, extracellular matrix-degrading enzymes, as well as by releasing reactive oxygen species into the tumour microenvironment (30). Indeed, some studies conducted using in vitro models have demonstrated that RET/PTC rearrangements and BRAF (V600E) mutation, the most frequent genetic events in PTC, can increase the expression of proinflammatory chemokines and cytokines, which facilitate tumour proliferation, migration and survival (32-34). However, it is worth mentioning that most studies have reported that RET/ PTC translocations are more frequent in PTC patients with HT than in patients with PTC alone, which has a higher frequency of BRAF (V600E) mutation in contrast $(35,36)$. Consistently, we found a significant and independent negative association between $B R A F$ (V600E) and HT in PTC patients. Because RET/PTC rearrangements is also detected in HT-affected thyroid epithelial cells (35), we believe that the molecular circuits linking HT and PTC mostly do not involve $B R A F$ (V600E) mutation, but preferably RET/PTC rearrangements.

Overall, we did not demonstrate a significant association between $B R A F(\mathrm{~V} 600 \mathrm{E})$ mutation and the remaining clinicopathological characteristics of PTC, which included sex, tumour size, histological subtype, multifocality, ETE, vascular invasion, lymph node and distant metastases, as well as intermediate/high risk to recurrence according to current guidelines. There were some important limitations in our study, notably the small sample size and the predominance of early-stage patients; therefore, we did not confirm the association between BRAF (V600E) mutation and aggressive behaviour and poor prognosis in PTC. The $8^{\text {th }}$ TNM edition recently removed microscopic ETE and regional lymph node metastasis from the classification of T3 disease. In addition, the $8^{\text {th }}$ TNM edition changed the age at diagnosis cut-off from 45 to 55 years, downstaging a significant number of PTC patients (37). Because we selected consecutive cases, only patients classified into the stage I/II were included in the present study. However, our results are in accordance with the majority of Brazilian studies, which did not find correlation between $B R A F$ mutations and PTC aggressive phenotype (15-20). In contrast, Oler and Cerutti reported that the BRAF (V600E) mutation was associated with tumour size, ETE, lymph node metastasis, high risk of recurrence and mortality in PTC patients (14). Many factors could explain this discrepancy, including case selection, different statistical approaches and the effect of possible confounding factors. Taking into account the conflicting results obtained from several studies addressing $B R A F(\mathrm{~V} 600 \mathrm{E})$ prognostic value in PTC around the world, some authors suggest reconsidering the clinical relevance of the $B R A F$ (V600E) mutation (10). Indeed, there is a possibility that this mutation may be in fact indirectly involved in tumour progression. By causing genomic instability, the $B R A F$ (V600E) mutation may coexist with secondary genetic and/or epigenetic alterations $(38,39)$, which in turn might cooperate in terms of tumour aggressiveness and even be more reliable prognostic indicators for PTC.

In conclusion, we found a high prevalence of $B R A F$ (V600E) mutation in a PTC case series from patients diagnosed and treated in Salvador, BA, Brazil. Furthermore, older age and concurrent HT was independently associated with the presence and absence of the $B R A F$ (V600E) mutation, respectively. We consider that further investigation is required to clarify potential mechanisms underlying the association between HT and the BRAF(V600E) mutation.

Acknownledgements: we would like to thank Dr. Edna Teruko Kimura for providing the protocol used to amplify the BRAF exon 15 . 
Funding statement: this work was supported in part by grants of FAPESB (Fundação de Amparo à Pesquisa no Estado da Babia, APP0040/2011).

Disclosure: no potential conflict of interest relevant to this article was reported.

\section{REFERENCES}

1. Bray F, Ferlay J, Soerjomataram I, Siegel RL, Torre L, Jemal A. Global cancer statistics 2018: GLOBOCAN estimates of incidence and mortality worldwide for 36 cancers in 185 countries. CA Cancer J Clin. 2018.

2. Lim H, Devesa SS, Sosa JA, Check D, Kitahara CM. Trends in thyroid cancer incidence and mortality in the United States, 1974-2013. JAMA. 2017;317(13):1338-48.

3. Noone AM, Howlader N, Krapcho M, Miller D, Brest A, Yu M, et al. (eds.). SEER Cancer Statistics Review, 1975-2015. Bethesda, MD. National Cancer Institute. Available from: https://seer.cancer.gov/ csr/1975_2015/.

4. Hirsch D, Levy S, Tsvetov G, Gorshtein A, Slutzky-Shraga I, Akirov $A$, et al. Long-term outcomes and prognostic factors in patients with differentiated thyroid cancer and distant metastases. Endocr Pract. 2017;23(10):1193-200.

5. Rosário PW, Ward LS, Carvalho GA, Graf H, Maciel RM, Maciel LM, et al.; Sociedade Brasileira de Endocrinologia e Metabologia. Thyroid nodules and differentiated thyroid cancer: update on the Brazilian consensus. Arq Bras Endocrinol Metabol. 2013;57(4):240-64.

6. Haugen BR, Alexander EK, Bible KC, Doherty GM, Mandel SJ, Nikiforov YE, et al. 2015 American Thyroid Association Management Guidelines for Adult Patients with Thyroid Nodules and Differentiated Thyroid Cancer: The American Thyroid Association Guidelines Task Force on Thyroid Nodules and Differentiated Thyroid Cancer. Thyroid. 2016;26(1):1-133.

7. Papaleontiou M, Haymart MR. New insights in risk stratification of differentiated thyroid cancer. Curr Opin Oncol. 2014;26(1):1-7.

8. Kimura ET, Nikiforova MN, Zhu Z, Knauf JA, Nikiforov YE, Fagin JA. High prevalence of BRAF mutations in thyroid cancer: genetic evidence for constitutive activation of the RET/PTC-RASBRAF signaling pathway in papillary thyroid carcinoma. Cancer Res. 2003;63(7):1454-7.

9. Xing M. BRAF mutation in papillary thyroid cancer: pathogenic role, molecular bases, and clinical implications. Endocr Rev. 2007;28(7):742-62.

10. Gandolfi G, Sancisi V, Piana S, Ciarrocchi A. Time to re-consider the meaning of BRAF V600E mutation in papillary thyroid carcinoma. Int J Cancer. 2015;137(5):1001-11.

11. Francis GL, Waguespack SG, Bauer AJ, Angelos P, Benvenga S, Cerutti JM, et al.; American Thyroid Association Guidelines Task Force. Management Guidelines for Children with Thyroid Nodules and Differentiated Thyroid Cancer. Thyroid. 2015;25(7):716-59.

12. Li C, Lee KC, Schneider EB, Zeiger MA. BRAF V600E mutation and its association with clinicopathological features of papillary thyroid cancer: a meta-analysis. J Clin Endocrinol Metab. 2012;97(12):4559-70.

13. Liu C, Chen T, Liu Z. Associations between BRAF(V600E) and prognostic factors and poor outcomes in papillary thyroid carcinoma: a meta-analysis. World J Surg Oncol. 2016;14(1):241.

14. Oler G, Cerutti JM. High prevalence of BRAF mutation in a Brazilian cohort of patients with sporadic papillary thyroid carcinomas: correlation with more aggressive phenotype and decreased expression of iodide-metabolizing genes. Cancer. 2009;115(5):972-80.
15. Araujo PP, Marcello MA, Tincani AJ, Guilhen AC, Morari EC, Ward LS. mRNA BRAF expression helps to identify papillary thyroid carcinomas in thyroid nodules independently of the presence of BRAFV600E mutation. Pathol Res Pract. 2012;208(8):489-92.

16. Dutenhefner SE, Marui S, Santos AB, de Lima EU, Inoue M, Neto JS, et al. BRAF: a tool in the decision to perform elective neck dissection? Thyroid. 2013;23(12):1541-6.

17. Penna GC, Pestana A, Cameselle JM, Momesso D, de Andrade FA, Vidal APA, et al. TERTp mutation is associated with a shorter progression free survival in patients with aggressive histology subtypes of follicular-cell derived thyroid carcinoma. Endocrine. 2018;61(3):489-98.

18. Pinheiro Dos Santos MJC, Bastos AU, da Costa VR, Delcelo R, Lindsey SC, Colozza-Gama GA, et al. LIMD2 Is Overexpressed in BRAF V600E-Positive Papillary Thyroid Carcinomas and Matched Lymph Node Metastases. Endocr Pathol. 2018;29(3):222-30.

19. da Silva RC, de Paula HS, Leal CB, Cunha BC, de Paula EC, Alencar RC, et al. BRAF overexpression is associated with BRAF V600E mutation in papillary thyroid carcinomas. Genet Mol Res. 2015;14(2):5065-75.

20. Lutz BS, Leguisamo NM, Cabral NK, Gloria HC, Reiter KC, Agnes $G$, et al. Imbalance in DNA repair machinery is associated with BRAFV600E mutation and tumour aggressiveness in papillary thyroid carcinoma. Mol Cell Endocrinol. 2018;472:140-8.

21. Kim HJ, Park HK, Byun DW, Suh K, Yoo MH, Min YK, et al. lodine intake as a risk factor for BRAF mutations in papillary thyroid cancer patients from an iodine-replete area. Eur $\mathrm{J}$ Nutr. 2018;57(2):809-15.

22. Campos Rde O, Reboucas SC, Beck R, de Jesus LR, Ramos YR, Barreto Idos $S$, et al. lodine Nutritional Status in Schoolchildren from Public Schools in Brazil: A Cross-Sectional Study Exposes Association with Socioeconomic Factors and Food Insecurity. Thyroid. 2016;26(7):972-9.

23. Veiga LH, Neta G, Aschebrook-Kilfoy B, Ron E, Devesa SS. Thyroid cancer incidence patterns in São Paulo, Brazil, and the U.S SEER program, 1997-2008. Thyroid. 2013;23(6):748-57.

24. Ito $Y$, Miyauchi A. Prognostic factors and therapeutic strategies for differentiated carcinomas of the thyroid. Endocr J. 2009;56(2):177-92.

25. Shen X, Zhu G, Liu R, Viola D, Elisei R, Puxeddu E, et al. Patient Age-Associated Mortality Risk Is Differentiated by BRAF V600E Status in Papillary Thyroid Cancer. J Clin Oncol. 2018;36(5): 438-45.

26. Vaisman F, Corbo R, Vaisman M. Thyroid carcinoma in children and adolescents-systematic review of the literature. J Thyroid Res. 2011;2011:845362.

27. Nikiforova MN, Ciampi R, Salvatore G, Santoro M, Gandhi M, Knauf JA, et al. Low prevalence of BRAF mutations in radiationinduced thyroid tumours in contrast to sporadic papillary carcinomas. Cancer Lett. 2004;209(1):1-6.

28. Liu D, Hu S, Hou P, Jiang D, Condouris S, Xing M. Suppression of BRAF/MEK/MAP kinase pathway restores expression of iodidemetabolizing genes in thyroid cells expressing the V600E BRAF mutant. Clin Cancer Res. 2007;13(4):1341-9.

29. Hiromatsu $Y$, Satoh H, Amino N. Hashimoto's thyroiditis: history and future outlook. Hormones (Athens). 2013;12(1):12-8.

30. Hanahan D, Weinberg RA. Hallmarks of cancer: the next generation. Cell. 2011;144(5):646-74.

31. Colotta F, Allavena P, Sica A, Garlanda C, Mantovani A. Cancerrelated inflammation, the seventh hallmark of cancer: links to genetic instability. Carcinogenesis. 2009;30(7):1073-81.

32. Russell JP, Shinohara S, Melillo RM, Castellone MD, Santoro M, Rothstein JL.Tyrosine kinase oncoprotein, RET/PTC3, induces the secretion of myeloid growth and chemotactic factors. Oncogene. 2003;22(29):4569-77. 
33. Borrello MG, Alberti L, Fischer A, Degl'innocenti D, Ferrario C, Gariboldi $\mathrm{M}$, et al. Induction of a proinflammatory program in normal human thyrocytes by the RET/PTC1 oncogene. Proc Natl Acad Sci U S A. 2005;102(41):14825-30.

34. Zhou D, Li Z, Bai X. BRAF (V600E) and RET/PTC Promote Proliferation and Migration of Papillary Thyroid Carcinoma Cells In Vitro by Regulating Nuclear Factor-kB. Med Sci Monit. 2017;23:5321-9.

35. Muzza M, Degl'Innocenti D, Colombo C, Perrino M, Ravasi E, Rossi $\mathrm{S}$, et al. The tight relationship between papillary thyroid cancer, autoimmunity and inflammation: clinical and molecular studies. Clin Endocrinol (Oxf). 2010;72(5):702-8.

36. Marotta V, Guerra A, Zatelli MC, Uberti ED, Di Stasi V, Faggiano A, et al. BRAF mutation positive papillary thyroid carcinoma is less advanced when Hashimoto's thyroiditis lymphocytic infiltration is present. Clin Endocrinol (Oxf). 2013;79(5):733-8.

37. Tuttle RM, Haugen B, Perrier ND. Updated American Joint Committee on Cancer/Tumor-Node-Metastasis Staging System for Differentiated and Anaplastic Thyroid Cancer (Eighth Edition): What Changed and Why? Thyroid. 2017;27(6):751-6.

38. Quiros RM, Ding HG, Gattuso P, Prinz RA, Xu X. Evidence that one subset of anaplastic thyroid carcinomas are derived from papillary carcinomas due to BRAF and p53 mutations. Cancer. 2005;103(11):2261-8.

39. Hou P, Liu D, Xing M. Genome-wide alterations in gene methylation by the BRAF V600E mutation in papillary thyroid cancer cells. Endocr Relat Cancer. 2011;18(6):687-97. 